\title{
The role of vestibular evoked myogenic potentials (VEMPs) in vestibulopathy diagnostics
}

\author{
Krempaska S, Koval J \\ University of P.J. Safarik, Faculty of Medicine, L.Pasteur University Hospital, Department of Otolaryngology \\ Head \&Neck Surgery, Košice, Slovakia. silviakrempaska@yahoo.com
}

\begin{abstract}
Objective: This study presents the role of vestibular evoked myogenic potentials (VEMPs) in the vestibular system investigation focused on the function of the saccule and inferior portion of the vestibular nerve. The main goal of the study is to report VEMP findings in different groups of patients with inner ear disorders symptoms and to identify some clinical application of this test in our patients.

Material and methods: WE have examined 218 patients with cochleovestibular symptomatology (sudden sensorineural cochlear hearing loss, retrocochlear hearing loss, vestibular neuronitis, benign paroxysmal positional vertigo (BPPV), Meniere's disease, central vestibular disorder, as well as vertigo of non vestibular ethiology and 32 patients with conductive hearing loss with VEMP testing. We performed I channel and II channels measurements. Results: We found no VEMP response in the group of conductive or mixed hearing loss in all cases $-100 \%$ of 32 patients, in sudden sensorineural cochlear hearing loss in $35.4 \%$ of 48 patients, in retrocochlear hearing loss in $19 \%$ of 26 patients, in vestibular neuronitis in $40 \%$ of 35 patients, in (BPPV) in $10 \%$ of 43 patients, in Meniere's disease in $61 \%$ of 18 patients, in non vestibular vertigo in no case of 22 patients and in central vestibular disorders in $35 \%$ of 23 patients.

Conclusion: The present study shows the potential value of VEMP investigation in periferal vestibulopathy diagnosis (Tab. 1, Fig. 7, Ref. 33). Full Text in PDF www.elis.sk.

Key words: VEMP, vestibulopathy, saccule, vestibulocollic reflex.
\end{abstract}

Dizziness and imbalance have become the most common reasons for patients to seek help from physicians. Many cases of dizziness result from causes other than vestibular dysfunction but, on the other hand, it is one of the most frequent symptoms in patients suffering from periferal vestibulopathy.

The ENT specialist usually performs an objective investigation of periferal sensory organs which helps him to localize the pathology and to qualify the function deficit. The vestibular system is not simple, it functions in a complex way, yet up to now still not fully explored. This might be a reason why its examination by objective methods is limited.

The investigation of vestibular function is generally based on both caloric and rotation tests when the ampullary receptors of the horizontal semicircular canal are stimulated (12, 25,3 2). To date, there has been no reliable clinical test of otolithic function. The vestibular normoreflexy is based on partial, not on complex examination of vestibular aparatus. We miss the valid information of vestibular functional status. As a result of this there is a group of patiens with vestibular normoreflexy and the vestibular diseases remain undiagnosed in the cases $(10,21)$.

University of P.J. Safarik, Faculty of Medicine, L.Pasteur University Hospital, Department of Otolaryngology Head \&Neck Surgery, Kosice, Slovakia

Address of correspondence: S. Krempaska, MD, Department of Otolaryngology Haed and Neck Surgery, UNLP a LF UPJS, Trieda SNP 1, SK-040 01 Kosice, Slovakia.

Phone: +421.55 .6403735$
Not long ago the peripheral vestibulopathy diagnostics have been enriched with objective investigation of otholitic organs using the vestibular evoked myogenic potentials method. Anatomic and physiologic studies have shown that the origin of the neural pathway is in the saccular macula. The response evoked by stimulated saccule is moving to the neurons of the Scarpa's ganglion and is transmited via the inferior vestibular nerve, through the inferior or lateral vestibular nucleus passing through the medial or lateral vestibulospinal tracts and finally gets to the motoneurons of the sternocleidomastoid muscles $(13,17,26,28)$.

Nowadays the investigation of the vestibulocollic reflex is a part of neurotologic clinical practice. Moreover this test evaluates not only functional integrity of otolithic organs but the functional integrity of inferior vestibular nerve as well $(3,4,16,23,29)$.

The aim of this study was to investigate the status of the vestibulospinal pathway in patients with inner ear symptoms, and to analyse the results in different types of vestibulopathies.

\section{Materials and methods}

Two hundred and fifty patients with inner ear symptoms were included into the study with age range of 18-73 years; 168 were women and 82 men.

All patients were examined by otomicroscopy, tympanometry, stapedial reflex examination, pure tone audiometry and electronystagmography (ENG). In some cases CT and/or MRI was indicated VEMPs were recorded ipsilaterally during acoustic stimulation. 


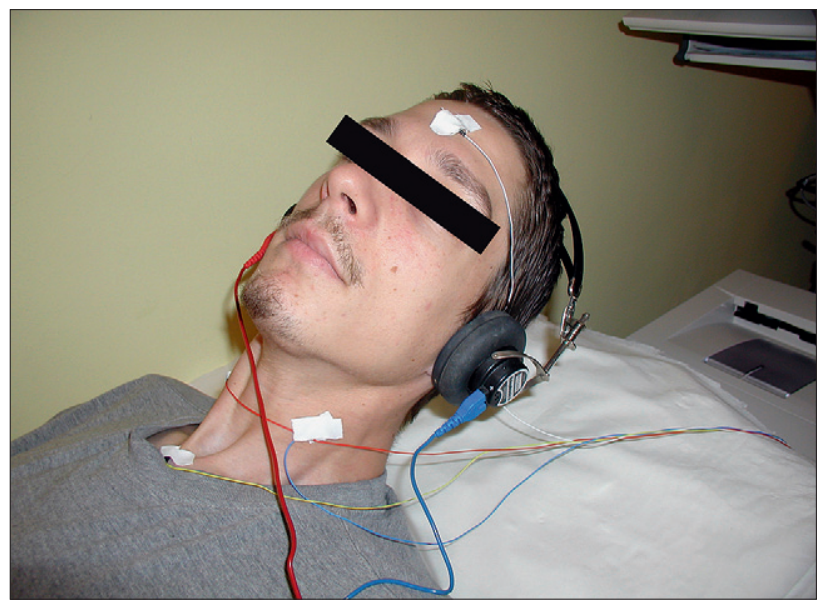

Fig. 1. Position of pacient during the VEMP test. Tone burst are delivered on the right side through a headphone.

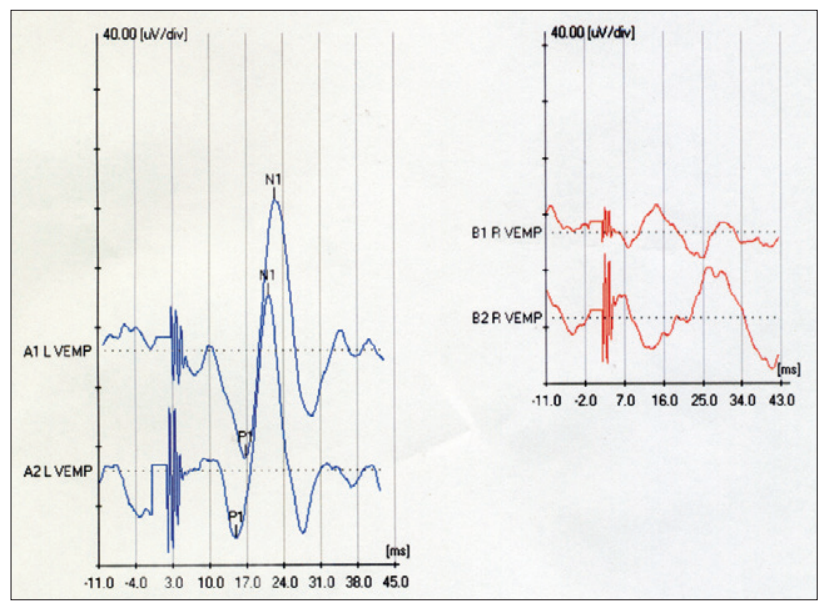

Fig. 2. VEMP asymetry in patient with right side retrocochlear hearing loss. MRI revealed right side acustic neurinoma.

The stimules (tone burst, intensity $100 \mathrm{~dB}$, frequency $1000 \mathrm{~Hz}$, stimulation rate $5 / \mathrm{s}$ ) were delivered by a Bio-logic Navigator PRO system, monoaurally using the Telephonics 296 headphones.

Electromyograph of the sternocleidomastoid muscle was recorded ipsilaterally by surface electrodes. Active electrodes were placed over the middle of the sternocleidomastoideus muscles on symetrical sides and the reference electrode over the upper sternum. The ground electrode was placed on the forehead. The subjects were in supine position. Before stimulation the patients had to elevate and turn their heads to the contralateral side and keep this position during the measurement to achieve a constant tonic activation of the sternocleidomastoid muscle (Fig. 1). The electromyographic signal was amplified, averaged, filtered and recorded. Two hundred responses were averaged for each measurement. We performed two consecutive measurements for valid reproductibility.

The patients were divided into eight groups (Tab. 1):

1. Conductive hearing loss -32 patients,

2. Acute sensorineural hearing loss -48 patients,
Tab. 1. VEMP findings in different patient's groups.

\begin{tabular}{lccc}
\hline Patients & $\begin{array}{c}\text { Normal } \\
\text { VEMP }\end{array}$ & IAD v A/L & $\begin{array}{c}\text { Absent } \\
\text { VEMP }\end{array}$ \\
\hline CHL & 0 & 0 & 32 \\
SSHL & 29 & $17 / 0$ & 2 \\
SHL - retrocochlear & 5 & 6 & 15 \\
VN ENG: PV & 5 & 0 & 16 \\
VN ENG:normal reaction & 8 & 0 & 6 \\
BPPV & 39 & $2 / 0$ & 2 \\
MD & 4 & $3 / 0$ & 11 \\
non vestibular vertigo & 22 & 0 & 0 \\
CND & 4 & $11 / 0$ & 8 \\
VNS & 0 & 0 & 3 \\
\hline
\end{tabular}

CHL - conductive hearing loss, SSHL - sudden sensorineural hearing loss, SHL sensorineural hearing loss, $\mathrm{VN}$ - vestibular neuronitis, ENG - elektronystagmography, PV - periferal vestibulopathy, BPPV - benign paroxysmal positional vertigo, MD - Meniere dissease, CND - central neural dissease, VNS - vestibular nerve section

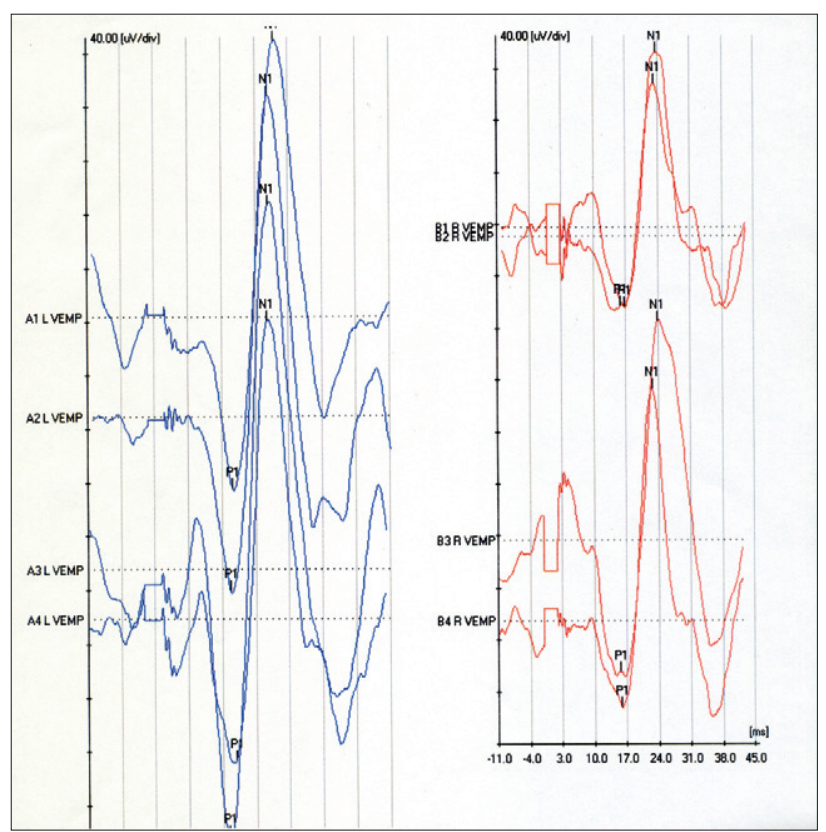

Fig. 3. Example of VEMP in the non vestibular vertigo group of patients. VEMP test revealed normal answer.

3. Retrocochlear sensorineural hearing loss (vestibular schwannoma) -26 patients,

4. Vestibular neuronitis -35 patients,

5. Benign paroxysmal positional vertigo -43 patients,

6. Meniere's disease -18 patients,

7. Vertigo of other ethiology (cardiovascular, hypotension, hypertension, psychogenic, hormonal disease, etc.) -22 patients,

8. Central vestibulopathy -23 patients.

\section{Results}

In the group of conductive and mixed hearing loss no VEMP response was detected.

Nearly all patients in the group of sensorineural hearing loss, a cochlear type, had a normal VEMP response. VEMPs were absent in two patients bilaterally and $35.4 \%$ of the patients had positive interaural amplitude differences. 


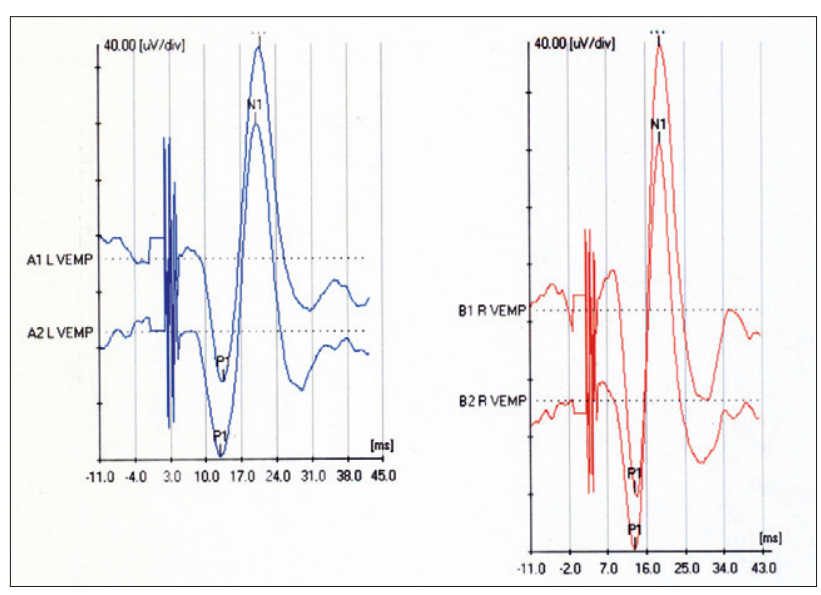

Fig. 4. VEMP test in patient suffering from acute right side sensorineural hearing loss without dizziness.

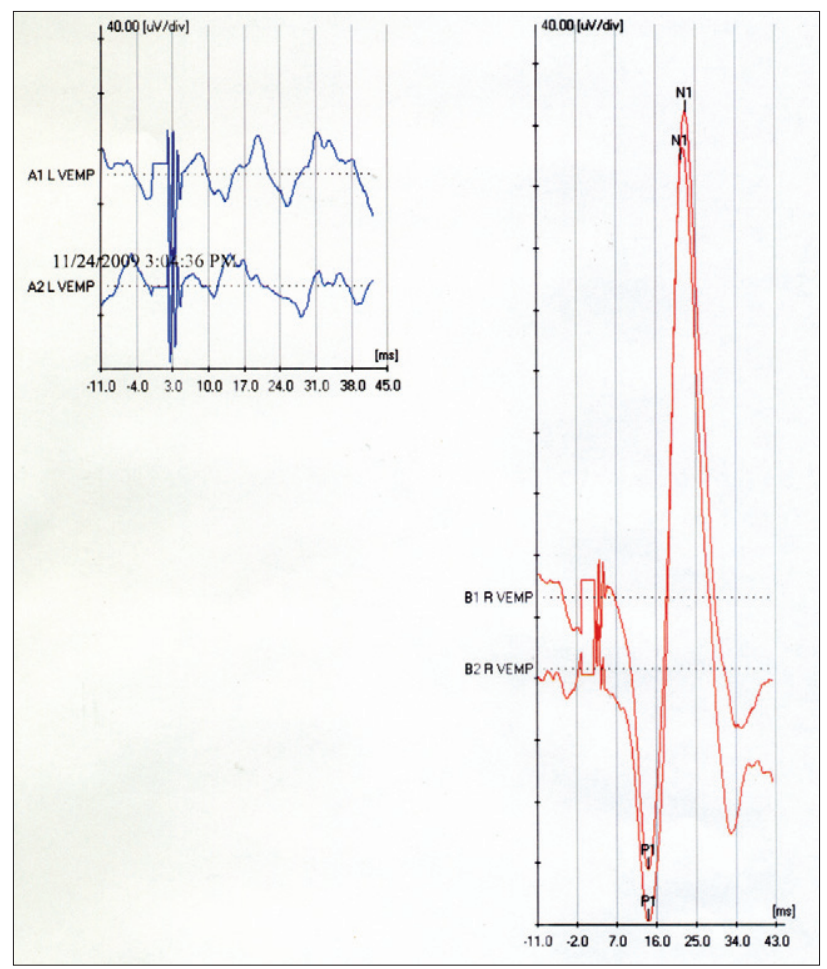

Fig. 5. VEMP obtained in patient with a left side conductive hearing loss. The VEMP on the left side absent, on the right was normal.

In the group of retrocochlear hearing loss $23 \%$ of patients showed reduced amplitude and/or prolonged latency. The VEMPs were not found in $57.7 \%$ and preserved in $19.2 \%$ (Fig. 2).

According to the ENG findings we divided the vestibular neuronitis group into two subgroups: the first one - the subgroup of patients with vestibular dysfunction on ENG, the second subgroup of patients with normoreflexy on ENG. In the first group VEMPs were preserved in $23.8 \%$ and in the other one in $57 \%$.

The VEMP response was normal in $90.7 \%$ in the benign position paroxysmal vertigo group (BPPV).

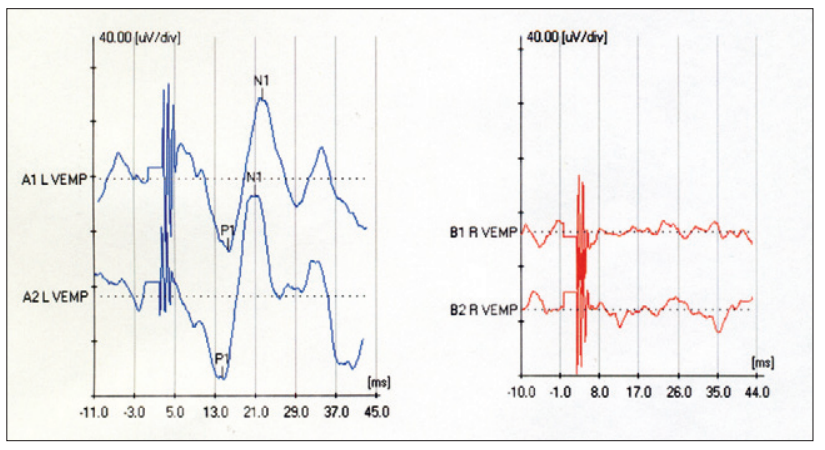

Fig. 6. VEMP recording of patient with right side vestibular neuronitis.

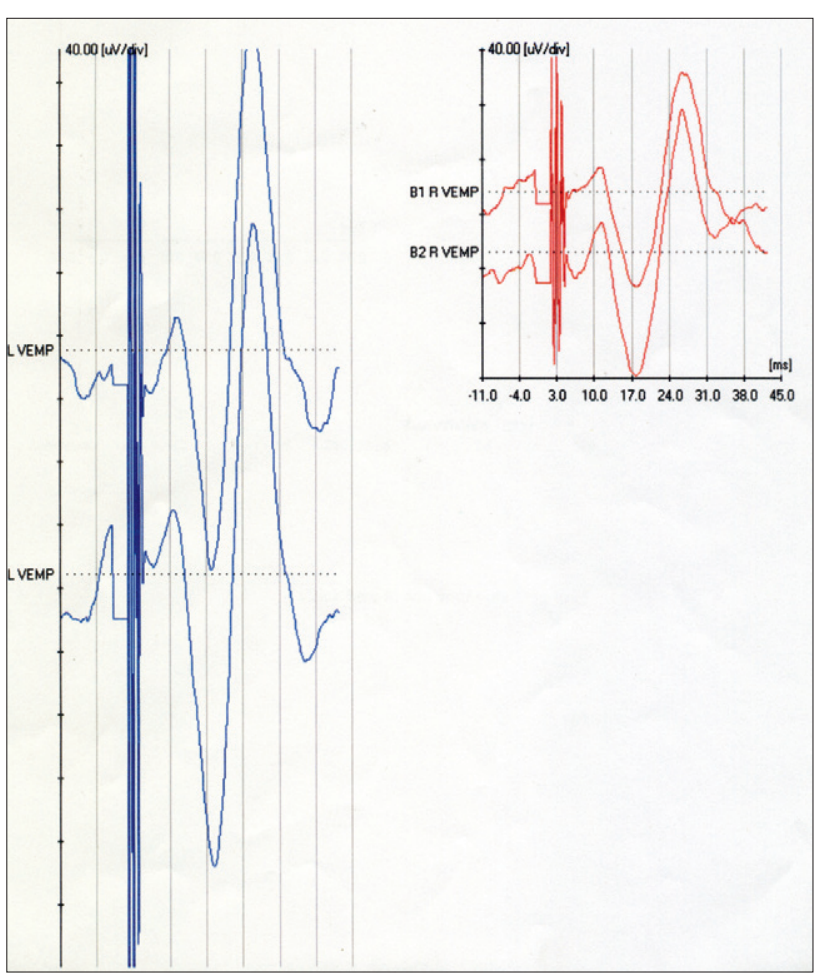

Fig. 7. VEMP of patient suffering from right side Menier's disease. Positive interaural amplitude difference.

In Meniere's disease group we have not registered VEMP response in $61 \%$. In $39 \%$ the response was present.

In the group of dizziness of non vestibular origin all patients had normal VEMPs (Fig. 3).

In the group of central vestibular diseases we obtained VEMP in $65.2 \%$ patients and in $34.8 \%$ VEMP was not found.

\section{Discussion}

Anatomically and phylogenically, the cochlear and the vestibular aparatuses are closely related. The sacculus is situated directly beneath the stapes footplate. Its localisation and relationship to the cochlear part of the inner ear is destined for the posibility of direct stimulation by lound sounds. 


\section{1-306}

During evolution the main function of the sacculus sensory organs had changed. Sacculus in humans has a specific role in balance but it has retained acoustic sensitivity, which is so specific for lower species $(9,11,25)$.

This knowledge and high stimulus intensity needed for stimulation may have induced uncertainty of the real VEMP origin. It was not clear if the response had been a result of preserved acoustic sensitivity of sacculus or a result of mechanic stimulation of vestibular sensory cells during the endolymph pressure changes.

Various studies of patiens with damage of the cochlear and vestibular organs have confirmed more vestibular than cochlear origin of the response $(1,12,22,25,33)$.

Later Cody and Bickford (6) and Todd and Cody (27) established vestibular origin of the response and identified the sacculus as a beginning of vestibulocollic reflex.

The cochlear origin of the reflex was described by Collebath and Halmagyi (7). Other authors investigated VEMP before and after vestibular nerve section $(21,33)$. They found absent VEMP after vestibular nerve section in all cases. We investigated VEMP in ten patients before and after vestibular nerve section. Since, no patients had VEMP response before surgery they have been excluded from our study.

Wu and Yang (31) evaluted the group of patients with sudden unilateral hearing loss without vertigo. They found VEMP in every case. The VEMP response in the same group of patiens was investigated and reported by numerous authors (33).

Our results in the group of sudden hearing loss are comparable with the above mentioned data (30). The VEMP response was present in the sensorineural group, cochlear type, nearly in all cases (Fig. 4). No correlation betwen the etiology, rate of formation and duration of hearing loss was found. In $35.4 \%$ of cases the amplitude changes typical for severe unilateral sensorineural hearing loss were found. In two cases VEPM was not recorded. In two patients over 60 years VEMPs were not recorded bilateraly, propably it was caused by decreased muscle contractibility.

It is generally known that conductive hearing loss prevents VEMP to be evoked, because the VEMP response is dependent upon a good transfer of sound from the external and middle ear to the inner ear. The responses are generally absent or reduced in conductive hearing loss in which the air-bone gap is $\geq 10$ 15 (30).

We investigated 20 subject with conductive or mixed hearing loss (Fig. 5). All patiens had no VEMP. Later we created the following criteria for investigation of patients with conductive hearing loss : negative otoscopy and higher intensity of dizziness in subjects exposed to loud sounds. That means, we investigated only subjects suspected of syndrom of superior semicircular canal dehiscence (SSCD).

VEMP testing is an ideal way to distinguish subjects with SSCD sydrome from otosclerosis. The third mobile window created by bone dehiscence over the semicircular canal increases transport of sound through the vestibulum and in this way the sacculus is activated by lower sound intensity. Patients have a vestibular hypersensitivity to sound stimulation causing a high VEMP amplitude and low threshold of response, intensity level around 55-70 dB (5, 20, 33).

The vestibular neuronitis is usually caused by viral infection. It may affect both superior vestibular nerve and inferior vestibular nerves. It happens that the infection causes inflamation of the entire vestibular ganglion $(8,23)$. The caloric vestibular test usually shows the unilateral ENG absent response after the stimulation of the lateral semicircular canal. That confirms the superior vestibular nerve neuronitis. On the other hand, the periferal vestibular dysfuncton in the superior vestibular nerve region is not necessary for vestibular neuronitis diagnostics. Althought the vestibular neuronitis in the inferior vestibular region is rare, the nerve might be affected separately and individually (Fig. 6). Murofushi et al (22) reported that one third of patients with vestibular neuronitis could have a disorder in the inferior region. The nerve damage is manifested with prolonged latency or with absent VEMP response (21). Our diagnosis of vestibular neuronitis was established by caloric and rotatory tests and standard neurootologic examination without VEMP. Only then we performed VEMP examination in all our patients. In the subgroup with superior vestibular nerve damage VEMPs were not found in $23.8 \%$ of patients. In the subgroup of vestibular normoreflexy VEMP coud not be found in $57.14 \%$ of patients. Viral affection of both superior and inferior vestibular nerves was supposed to occure in these subjects. So exact diagnosis without examination of vestibulocollic reflex is not possible.

The lack of clinical tests for investigation of the posterior semicircular canal or saccular function leads to a number of misdiagnosed patients with vestibular neuronitis in the inferior region. Consequently, the patients with normoreflexy in rotatory and caloric tests and without VEMP testing are usually considered to have a central vestibular disease.

In cases with vestibular neuronitis of superior vestibular nerve the VEMP responses are normal or nearly normal. When following these patients they can be later found to suffer from benign positional paroxysmal vertigo. In spite of the fact, that it can be a sequel of the disease, the exact mechanism for canalolithiasis is not clear yet. Numerous papers documented normal VEMP results in the BPPV patients. Only $5 \%$ of our BPPV patients had no VEMP response and another 5\% showed only interaural amplitude latency difference. Our findings are in line with the literature (1). These findings of BPPV patinets although interesting need further observation.

Meniere's disease is characterized by fluctuating hearing loss, tinnitus, aural fullness and rotatory vertigo. Etiology is unknown, but histopathologic examinations reveal endolymphatic hydrops of the affected labyrinth. Endolymphatic hydrops may damage hair cells most often in the cochlea, then followed by saccule, utricule and semicircular canals $(1,2,18,19)$. De Waele et al (8) found that the patiens suffering from Meniere's disease had no VEMP responses in $54 \%$ of cases. Comparable results were published by Shojaku et al (33). The authors concluded that the VEMP testing could provide important information on the state of the disease.

The VEMP can be normal or with increased/decreased amplitude depending on fluctuating hearing due to saccular distension. 
Normal VEMP responses after Meniere's attack indicate that the hair cells of the saccular macula remain intact throughout the attack. On the other hand, depressed or absent VEMPs may represent a collapse of the saccule (Fig. 7). VEMPs may or may not be normalized after the attack, depending on the functional state of the saccule.

In $61 \%$ of our Meniere's disease patients VEMPs were not found and in $39 \%$ they were present. These findings correlate with the literature $(1,2,8,10,18,19,33)$, and indicate that there are a lot of patients having pathologic VEMP responses, e.i. a contribution of sacullar damage may be supposed in the Meniere's disease, as well.

The diagnosis of Meniere's disease is based on symptomathology, glycerol test, electrocochleography (ECoG), ENG, CHAMP ERA (cochlear hydrops analysis masking procedure). In clinical application these tests determine the status and severity of the Meniere's disease. The interaural difference is the most reliable indicator. Although there is an increasing number of data on the VEMP testing in Meniere's disease, we agree that it still remains limited in effective clinical use in Meniere's disease compared with the other methods .

Pathologic VEMP responses were found in patiens with central nervous disorders (14). Absent or delayed latency can be observed in patients with basilar type of migraine. It is supposed that the problem is due to disrupted functional integrity of a neural pathway at the brainstem level. Some patients suffering from sclerosis multiplex had prolonged latency of VEMP, which may be caused by a demyelinisation conduction block and consequent axon degeneration in the vestibulospinal tract (24). VEMPs are missing in the spinocerebellar degeneration diseases because of the the periferal vestibular way disability. Pathologic VEMPs have been found in patients after sudden cerebellar attack and in all types of cerebellopontine angle tumours compressing the cochleovestibular nerve. There is not enough information concerning VEMPs in the central nervous disorders. We need further studies to precise the clinical application of VEMP in this group of patients.

\section{Conclusion}

The advantages of VEMP testing compared with other methods in clinical practice are following:

VEMP test is relatively easy to perform and lasts a very short time. The investigation is tolerated in all age groups of patients very well.

VEMP test is highly specific in locating a lesion and migth also provide information on the saccular pathology and inferior vestibular nerve dysfunction.

VEMP test is very sensitive and able to reveal minimal changes in the vestibular function.

The study confirms the necessity of VEMP in clinical neurotologic application. It is a new way of assessing the vestibular system and the results may influence the diagnosis of vestibular pathologies.

\section{References}

1. Akkuzu G, Akkuzu B, Ozluoglu LN. Vestibular evoked myogenic potentials in benign paroxysmal positional vertigo and Meniere's disease. Eur Arch Otorhinolaryngol 2006; 263: 510-517.

2. Ban JH, Lee JK, Jin SM et al. Glycerol pure tone audiometry and glycerol vestibular evoked myogenic potential: representing specific status of endolymphatic hydrops in the inner ear. Eur Arch Otorhinolaryngol 2007; 264: 1275-1281.

3. Basta D, Todt I, Ernst A. Characterization of age- related changes in vestibular evoked myogenic potentials. J Vest Res 2007; 17: 93-98.

4. Beyea JA, Zeitouni AG. Vestibular evoked myogenic potentials in healthy control subjects using the head rotation method. J Otolaryngol Head Neck Surg 2008; 37: 522-527.

5. Brantberg K, Granath K, Schart N. Age-Related changes in vestibular evoked myogenic potentials. Audiol Neurotol 2007; 12: 247-253.

6. Cody D, Bickford R. Average evoked myogenic responses in normal man. Laryngoscope 1969; 79: 400-446.

7. Colebatch JG, Halmagyi GM. Vestibular evoked potentials in human neck muscles before ang after unilateralvestibular deafferantation. Neurology 1992; 42: 1635-1636.

8. De Waele C, Huy PT, Diard JP et al. Saccular dysfunction in Meniere's disease. Am J Otol 1999; 20: 223-232.

9. Eleftheriadou A, Defterreos SN, Zarikas V et al. Vestibular myogenic potential eliciting in normal subjects: comparison of four different metods. J Otolaryngol HeadNeck Surg 2008; 37: 704-711.

10. Faralli M, Molini E, Ricci G et al. Study of Vestibular evoked myogenic potentials in unilateral vestibulopathy: Otolithic versus canal function testing. Otol Neurotol 2006; 27: 1115-1119.

11. Chen CHN, Wang SJ, Wang CT et al. Vestibular evoked myogenic potentials in newborns. Audiol Neurotol 2007; 12: 59-63.

12. Isaacson B, Murphy E, Cohen H. Does the metod of sternocleidomastoid muscle activation affect the vestibular evoked myogenic potential response? J Vest Res 2006; 16: 187-191.

13. Ito K, Karina S, Murofushi T. Effect of head position on vestibular evoked myogenic potentials with toneburst stimuli. Acta Otolaryngol 2007; 107: 57-61.

14. Jacot E, Wiener-Vacher S. Potential value of vestibular evoked myogenic potentials in paediatric neuropathies. J Vestib Res 2008; 18 : 231-237.

15. Krempaská S. Vestibulárne evokované myogénne potenciáli-VEMP: štandardizácia metódy. Otorinolaryng a Foniat (Prague) - in press.

16. Lee KJ, Kim MS, Son EJ et al. The usefulness of rectified VEMP. Clin Exp Otolaryngol 2008; 1: 143-147.

17. Lee SK, Cha CHI, Jung TS et al. Age-related differences in parameters in parameters of vestibular evoked myogenic potentials. Acta OtoLaryngol 2008; 128: 66-72.

18. Magliulo G, Parrotto D, Gagliardi S et al. Vestibular evoked periocular potentials in Meniere'sdisease after glycerol testing. Ann Otol Rhinol Laryngol 2008; 117: 800-804.

19. Magliulo G, Cianfrone G, Gagliardi M et al. Vestibular evoked myogenic potentials and distortion-product otoacoustic emissions combined with glycerol testing in endolymphatic hydrops: their value in early diagnosis. Ann Otol Rhinol Laryngol 2004; 113: 1000-1005. 


\section{1-306}

20. Modugno GC, Magnani G, Brandolini C et al. Could vestibular evoked myogenic potentials (VEMPs) also be useful in the diagnosis of perilymphatic fistula? Eur Arch Otorhinolaryngol 2006; 263: 552-555.

21. Monstad P, Okstad S, Mygland A. Inferior vestibular neuritis: 3 cases with clinical features of acute vestibular neuritis, normal calorics bud indications of saccular failure. BMC Neurology 2006; 45: 2-6.

22. MurofushiT, Ochiai A, Ozeki H et al. Laterality of vestibular evoked myogenic potentials. Int J Audiol 2004; 43: 66-68.

23. Ozeki H, Iwasaki S, Ushio M et al. The lesion of vestibular dysfunction in Ramsay Hunt syndrome: A study by click and galvanic VEMP. J Vest Res 2006; 16: 217-222.

24. Patko T, Simo M, Arányi Z. Vestibular clic- evoked myogenic potentials: sensitivity and factors determining abnormality in patients with multiple sclerosis. Multiple Sclerosis 2007; 13: 193-198.

25. Shinjo Y, Jin Y, Kaga K. Assesment of vestibular function of infants and children with congenital and acquired deafness using the ice-water caloric test,rotation chair test vestibular-evoked myogenic, potential recording. Acta OtoLaryngol 2007; 127: 736-747.

26. Tal D, Hershkovitz D, Kaminski G et al. Vestibular evoked myogenic potential threshold and seasickness susceptibility. J Vestib Res 2006; 16: $273-278$
27. Todd NP, Cody FWJ, Banks JR. A saccular origin of frekvency tunning in myogenic vestibular evoked potentials?Implications for human responses to loud sounds. Hearing Res 2000; 141: 180-188.

28. Trivelli M, Vicini C, $\mathbf{D}^{\prime}$ Ascanio $\mathbf{L}$ et al. The effect of logon versus click on vestibular evoked myogenic potentials. Acta OtoLAryngol 2008; 128: 314-317.

29. Vanspauwen R, Wuyts FL, Van de Heyning PH. Improving Vestibular Evoked Myogenic Potential Reliability by using a blood pressure manometer. Laryngoscope 2006; 116: 131-135.

30. Wang MCH, Lee GS. Vestibular evoked myogenic potentials in middle ear effusion. Acta OtoLaryngol 2007; 127: 700-704.

31. Wu CH, Young YH, Murofushi T. Tone burst-evoked myogenic potentials in human neck flexor and extensor. Acta Otolaryngol 1999; 119: $741-744$

32. Young YH. Vestibular evoked myogenic potentials: optimal stimulation and clinical application. J Biomed Sci 2006; 13: 745-751.

33. Zhou G, Cox LC. Vestibular evoked myogenic potentials: History and Overview. Am J Audiol 2004; 13: 135-143.

Received October 5, 2010. Accepted February 14, 2012. 